\title{
Fetal Acrania (Exencephaly) in the Context of a Pregnant Female Taking Adalimumab for Psoriasis: A Case Report
}

This article was published in the following Dove Press journal: Biologics: Targets and Therapy

Nujud Daham (D)'

Abdullah AIMuqrin (1D) ${ }^{2}$

Abdulaziz Madani ${ }^{3}$

Fahad AISaif ${ }^{3}$

'Department of Dermatology, Dr. Sulaiman Al-Habib Medical Group, Riyadh, Saudi Arabia; ${ }^{2}$ Department of Dermatology, Prince Mohammed Bin Abdulaziz Medical City, Riyadh, Saudi Arabia; ${ }^{3}$ Department of Dermatology, College of Medicine, King Saud University, Riyadh, Saudi Arabia
Correspondence: Nujud Daham Department of Dermatology, Dr. Sulaiman Al-Habib Medical Group, King Fahad Road, Olaya, Po Box 91877, Riyadh I I643, Saudi Arabia

Tel +9665002III 34

Email nujud.daham@gmail.com

\begin{abstract}
Adalimumab is a fully human, recombinant, IgG1 monoclonal antibody that targets tumor necrosis factor-alpha (TNF-alpha). It has been established that adalimumab can cross the placenta and can be detected in the fetal circulation for up to 6 months postpartum. However, clinical studies have failed to show any consistent or specific adverse fetal outcomes from maternal exposure to adalimumab during pregnancy. In our report, we present a case of fetal acrania (exencephaly) in the setting of a pregnant female taking adalimumab prior to and during pregnancy. Exencephaly is a neural tube defect (NTD) that results from failure of closure of the neural fold. It is true that there were other risk factors that might have contributed to our patient's unfortunate outcome. For example, she did not take folic acid supplementation prior to or during her pregnancy. Nonetheless, studies have shown that folic acid deficiency alone is not sufficient to lead to the development of NTDs. Our patient's exposure to adalimumab during her pregnancy might have added to the risk in her situation. Our report aims to inform clinicians of that possible risk and to stimulate them to report any similar outcomes.
\end{abstract}

Keywords: adalimumab, exencephaly, neural tube defects, pregnancy, psoriasis, TNF- $\alpha$

\section{Introduction}

Adalimumab is a fully human, recombinant, IgG1 monoclonal antibody that targets with specificity tumor necrosis factor-alpha (TNF- $\alpha) .{ }^{1}$ According to the 1979 FDA pregnancy risk categories, adalimumab is classified as pregnancy category $\mathrm{B}$ and appears to be safe to use by pregnant women during the first and second trimesters. $^{2,3}$ In 2015, the FDA replaced these categories by the Pregnancy and Lactation Labeling Rule (PLLR) which requires drug companies to present more information to providers and patients. ${ }^{4}$ Guidelines published by the British Society for Rheumatology and the British Health Professionals in Rheumatology in 2016 recommended discontinuing adalimumab at the end of the second trimester of pregnancy. ${ }^{5}$ However, this was mainly to avoid high drug levels in cord blood at delivery and the need to refrain from giving live vaccines to infants younger than 7 months old. ${ }^{5}$ Nonetheless, the association between adalimumab and poor pregnancy outcomes continues to be a matter of debate. Despite the existence of research studies addressing this concern, data provided by such studies are limited and the recommendations given are inconclusive. ${ }^{6,7}$ We present a case of fetal acrania (exencephaly) which led to the termination of pregnancy in the setting of 
a pregnant female taking adalimumab prior to and during pregnancy for treatment of psoriasis.

\section{Case Report}

A 24-year old female (G1P0A1) was diagnosed with psoriasis at the age of four. It was fairly controlled until the end of year 2015 when she presented with multiple guttate scaly papules and plaques affecting the extensor aspects of her limbs as well as her scalp. Total body surface area (BSA) involvement approached 15\%. An $\mathrm{x}$-ray was performed at that time which did not show joint involvement. The decision was made to start the patient on adalimumab. Prior to initiating the treatment, laboratory testing, in addition to posteroanterior (PA) and lateral chest $\mathrm{x}$-rays were performed. All results were within normal limits. Therefore, on February 8, 2016, the patient was started on adalimumab. Her psoriasis was well controlled until September 3, 2018, when she presented with new psoriatic lesions. At the time of her presentation, she was 5 weeks pregnant. Her body mass index (BMI) was 25.6. The patient was evaluated by the on-call team who recommended that she continues taking adalimumab. Topical mometasone furoate was also prescribed to be applied twice daily. On November 19, 2018, the patient presented to the dermatology department for a follow-up and at that time, her psoriasis was well controlled. Unfortunately, she informed our team that she had received a prenatal diagnosis of fetal exencephaly. The patient stated that she had not been taking folic acid prior to or during her pregnancy. Also, no maternal folic acid level was obtained. Maternal glucose levels were within normal limits. She initially denied consanguinity. However, upon further questioning, she stated that she is indeed married to her first cousin. Nonetheless, she denied a history of a similar diagnosis in any of her or her husband's family members. She also provided a history of an upper respiratory tract infection one week prior to learning of her pregnancy. She was evaluated at a private hospital, where her fever was documented to be 40 degrees Celsius. After receiving 1 dose of IV paracetamol and 1 dose of an IV antibiotic, she was discharged on oral amoxicillin/clavulanic acid. Based on the history she had given, our team made the decision to immediately refer the patient to the obstetrics department and recommended that she continues her treatment with adalimumab. She underwent an obstetric ultrasound, which again, confirmed the diagnosis of fetal exencephaly. Since the condition is considered incompatible with life, the patient was presented with an option to terminate her pregnancy which she accepted. She was given a total of 5 vaginal doses of misoprostol, $400 \mathrm{mcg}$ each. The patient passed the fetus and placenta on Nov 23, 2018.

\section{Discussion}

Exencephaly is a neural tube defect (NTD) that results from the failure of closure of the neural fold. ${ }^{8}$ Multiple studies have proven the protective effect of folic acid supplementation in pregnancy and its role in preventing NTDs. ${ }^{8}$ The fact that our patient did not receive folic acid supplementation prior to or during her pregnancy could explain the consequence. However, in most cases, even without folic acid supplementation, maternal folate levels remain within normal limits. ${ }^{8}$ Furthermore, folate deficiency is not the sole contributor to the occurrence of NTDs. In fact, most NTDs in humans are multifactorial, involving both genetic and environmental factors ${ }^{8}$. For example, the association between NTDs and prenatal exposure to valproic acid has been well established. ${ }^{8}$ Additionally, both maternal obesity (BMI $\geq 30$ ) and diabetes mellitus can increase the risk of NTDs. ${ }^{9}$ A review article by Imbard et al suggests that NTDs result not from folic acid deficiency alone, but from the interaction between folic acid deficiency and other individually insufficient factors. ${ }^{10}$ Therefore, we can not rule out the role played by adalimumab in causing this unfavorable outcome. Furthermore, being an IGg1 antibody, adalimumab can actively cross the placenta and is detectable in cord blood and infants' serum up to 7 months after birth. ${ }^{11}$ Nevertheless, it is still unclear how such an exposure may adversely affect pregnancy outcomes. ${ }^{11}$ Animal studies suggest that TNF- $\alpha$ plays a role in embryogenesis. The use of TNF- $\alpha$ inhibitors may interfere with this role ${ }^{12}$. A prospective multi-center cohort study by WeberSchoendorfer et al compared 495 pregnancies exposed to TNF- $\alpha$ inhibitors to 1532 non-exposed pregnancies. This study revealed that the risk of birth defects is higher in the exposed group than it is in the comparison group. However, no specific birth defects were identified to be associated with the use of TNF- $\alpha$ inhibitors. ${ }^{12}$ A concise report by Burmester et al concluded that there is no difference in birth defect frequencies between pregnant women with rheumatoid arthritis (RA) exposed to adalimumab, pregnant women with RA not exposed to adalimumab and otherwise healthy pregnant women. Moreover, no specific pattern was detected among the reported birth defects. $^{13}$ 


\section{Conclusion}

We cannot confirm the causality between the use of adalimumab and the occurrence of exencephaly in this case. Our report, however, may stimulate other clinicians to report similar outcomes. This can aid in overcoming some of the controversy surrounding the safety of using adalimumab during pregnancy.

\section{Abbreviations}

TNF- $\alpha$, tumor necrosis factor alpha; FDA, Food and Drug Administration; PLLR, Pregnancy and Lactation Labeling Rule; BSA, body surface area; PA, posteroanterior; BMI, body mass index; IV, intravenous; NTDs, neural tube defects; RA, rheumatoid arthritis.

\section{Ethics and Consent}

A written informed consent has been obtained from the patient to publish case details. No institutional approval was required to publish case details.

\section{Funding}

This article has no funding source.

\section{Disclosure}

The authors have no conflicts of interest to declare.

\section{References}

1. Menter A, Tyring SK, Gordon K, et al. Adalimumab therapy for moderate to severe psoriasis: a randomized, controlled Phase III trial. J Am Acad Dermatol. 2008;58:106-115. doi:10.1016/j.jaad.2007.09.010

2. Puchner A, Gröchenig HP, Sautner J, et al. Immunosuppressives and biologics during pregnancy and lactation. Cent Eur J Med. 2019;131:29-44.
3. Skorpen CG, Hoeltzenbein M, Tincani A, et al. The EULAR points to consider for use of antirheumatic drugs before pregnancy, and during pregnancy and lactation. Ann Rheum Dis. 2016;75:795-810. doi:10.1136/annrheumdis-2015-208840

4. Pernia S, De Maagd G. The new pregnancy and lactation labeling rule. Pharm Ther. 2016;41:713-715.

5. Flint J, Panchal S, Hurrell A, et al. BSR and BHPR guideline on prescribing drugs in pregnancy and breastfeeding-Part I: standard and biologic disease modifying anti-rheumatic drugs and corticosteroids. Rheumatology. 2016;55:1693-1697. doi:10.1093/rheumatology/ kev404

6. Julsgaard M, Christensen LA, Gibson PR, et al. Concentrations of adalimumab and infliximab in mothers and newborns, and effects on infection. Gastroenterology. 2016;151:110-119. doi:10.1053/j. gastro.2016.04.002

7. Johansen CB, Jimenez-Solem E, Haerskjold A, et al. The use and safety of TNF inhibitors during pregnancy in women with psoriasis: a review. Int J Mol Sci. 2018;19:1349. doi:10.3390/ijms19051349

8. Greene NDE, Copp AJ. Neural tube defects. Annu Rev Neurosci. 2014;37:221-242. doi:10.1146/annurev-neuro-062012-170354

9. Lupo PJ, Canfield MA, Chapa C, et al. Diabetes and obesity-related genes and the risk of neural tube defects in the national birth defect prevention study. Am $J$ Epidemiol. 2012;176:1101-1109. doi:10.1093/aje/kws 190

10. Imbard A, Benoist J-F, Blom HJ. Neural tube defects, folic acid and methylation. Int J Environ Res Public Health. 2013;10:4352-4389. doi:10.3390/ijerph10094352

11. Van derWoude CJ, Ardizzone S, Bengtson MB. et al. ECCO Guidelines/Consensus Paper. The Second European Evidenced-Based Consensus on Reproduction and Pregnancy in Inflammatory Bowel Disease. J Crohns Colitis. 2015:107-124. doi:10.1093/ecco-jec/jju006

12. Weber-Schoendorfer C, Oppermann M, Wacker E, et al. Pregnancy outcome after TNF- $\alpha$ inhibitor therapy during the first trimester: a prospective multicentre cohort study. $\mathrm{Br} J$ Clin Pharmacol. 2015;80:727-739. doi:10.1111/bcp.12642

13. Burmester GR, Landewé R, Genovese MC, et al. Adalimumab long-term safety: infections, vaccination response and pregnancy outcomes in patients with rheumatoid arthritis. Ann Rheum Dis. 2017;76:414-417. doi:10.1136/annrheumdis-2016-209322
Biologics: Targets and Therapy

\section{Publish your work in this journal}

Biologics: Targets and Therapy is an international, peer-reviewed journal focusing on the patho-physiological rationale for and clinical application of Biologic agents in the management of autoimmune diseases, cancers or other pathologies where a molecular target can be identified. This journal is indexed on PubMed Central, CAS, EMBase,
Scopus and the Elsevier Bibliographic databases. The manuscript management system is completely online and includes a very quick and fair peer-review system, which is all easy to use. Visit http://www.dovepress.com/testimonials.php to read real quotes from published authors. 